\title{
Minimally invasive surgery for gastric cancer: the American experience
}

\author{
Erin K. Greenleaf ${ }^{1} \cdot$ Susie X. Sun ${ }^{1}$ Christopher S. Hollenbeak ${ }^{1,2}$ • \\ Joyce Wong ${ }^{1}$
}

Received: 18 November 2015/Accepted: 22 February 2016/Published online: 9 March 2016

(c) The International Gastric Cancer Association and The Japanese Gastric Cancer Association 2016

\begin{abstract}
Background Minimally invasive surgical techniques are increasingly being implemented in oncologic care. This study assesses the impact of minimally invasive surgery on oncologic and perioperative outcomes in the management of gastric cancer in the USA.

Methods From the American College of Surgeons and American Cancer Society National Cancer Data Base, we identified 6427 patients who underwent gastrectomy for cancer from 2010 to 2012. Treatment groups were categorized with an intention-to-treat paradigm as robotic, laparoscopic, and open surgery. Univariate and multivariate analyses were performed to estimate the impact of the surgical approach on oncologic and perioperative outcomes.

Results Of patients undergoing definitive surgical intervention, $3.5 \%(n=223)$ underwent robotic gastrectomy, $23.1 \%(n=1487)$ underwent laparoscopic gastrectomy, and $73.4 \%(n=4717)$ underwent open surgery. Minimally invasive gastrectomy was more frequently
\end{abstract}

Disclaimer The National Cancer Data Base (NCDB) is a joint project of the Commission on Cancer $(\mathrm{CoC})$ of the American College of Surgeons and the American Cancer Society. The CoC's NCDB and the hospitals participating in the CoC's NCDB are the source of the deidentified data used herein; they have not verified and are not responsible for the statistical validity of the data analysis or the conclusions derived by the authors.

Joyce Wong

jwong1@hmc.psu.edu

1 Department of Surgery, The Pennsylvania State University College of Medicine, 500 University Drive, H070, Hershey, PA 17033-0850, USA

2 Department of Public Health Sciences, The Pennsylvania State University College of Medicine, Hershey, PA, USA performed on white $(P=0.018)$, privately insured patients $(P=0.049)$ treated at academic centers $(P<0.0001)$ in the eastern USA $(P<0.0001)$. After demographics, comorbidities, and tumor-related factors had been controlled for, patients who underwent laparoscopic gastrectomy had the postoperative length of stay decreased by 1.08 days $(P<0.0001)$ and greater odds of having at least 15 lymph nodes resected (odds ratio $1.16, P=0.023$ ). Use of robotic surgery did not have a statistically significant effect on the postoperative length of stay relative to open surgery $(P=0.222)$ but the patients so treated had greater odds of having at least 15 lymph nodes resected (odds ratio $1.51, P=0.005)$. There were no differences in $\mathrm{R} 0$ resection rates or perioperative mortality on the basis of the surgical approach alone.

Conclusions These findings suggest that use of minimally invasive surgery for gastric cancer in the USA is impacting the adequacy of oncologic resection but is not yet having a clinically significant impact on perioperative outcomes relative to a conventional open approach.

Keywords Minimally invasive - Gastrectomy - Gastric cancer - Outcomes

\section{Introduction}

Since the advent of laparoscopic surgery in the twentieth century, minimally invasive approaches are now commonly used techniques for general surgery procedures. Minimally invasive surgery already has a strong foothold in the realm of urologic and gynecologic surgery. More recently, the field of surgical oncology has begun to adopt minimally invasive options in the surgical treatment of patients with malignancy [1]. Building on a foundation of 
procedures performed laparoscopically in bariatrics, as well as in the management of splenic, diaphragmatic, and other foregut-related problems, Kitano et al. [2] reported the first laparoscopically assisted Billroth I gastrectomy for early gastric cancer in 1994 [1]. Over the last 20 years, the safety and oncologic efficacy of laparoscopic gastrectomy has been studied, perhaps most notably by the Korean Laparoscopic Gastrointestinal Surgery Study (KLASS) Group and the Japanese Laparoscopic Surgery Study Group (JLSSG) [3-6]. The recent implementation of robotic technology has further added to the armamentarium of surgical oncologists, providing a corresponding ease with which surgical dissections are performed [7-9].

The benefits of minimally invasive surgical methods are proven and well published [6, 10-16]. With advantages such as decreased postoperative pain and shorter length of hospital stay, minimally invasive techniques are heralded as a significant development in the progress of general surgery. However, minimally invasive surgery is not without its detractors, who cite added operative time and associated costs, particularly with use of a robotic technique, as limitations [5]. Whether the technical advantages offset the progressively diminishing drawback of cost has yet to be seen in the setting of gastric cancer treated in the USA, as the predominance of this disease, and the literature related to it, is studied from an Eastern perspective.

Because US gastric cancer care is not centralized, unlike in Eastern countries, and patients come from an especially heterogeneous demographic background with varied medical coverage, this study was undertaken to examine practice patterns across the USA for patients with surgically resectable gastric cancer and to assess the adequacy of oncologic resection and consequent perioperative outcomes. Specifically, our aim was to estimate trends in the use of minimally invasive approaches and to compare patients who underwent robotic, laparoscopic, and open gastrectomy with regard to surgical margins and lymph node retrieval for the adequacy of oncologic resection, as well as length of stay (LOS) and short-term mortality for perioperative outcomes. We hypothesized that patients who underwent robotic and laparoscopic surgery would have a statistically significant improvement in oncologic resection and LOS, but no difference in postoperative mortality.

\section{Methods}

\section{Data}

This study was a retrospective cohort study using tumor registry data collected between 2003 and 2012 in the National Cancer Data Base (NCDB), a joint effort by the Commission on Cancer of the American College of
Surgeons and the American Cancer Society. After approval had been secured in December 2014, access was obtained to this database, which captures approximately $80 \%$ of the cancer cases in the USA.

Our study population was restricted to patients who underwent definitive gastrectomy for gastric cancer. Definitive gastrectomy was defined as proximal gastrectomy, distal gastrectomy, total gastrectomy, and gastrectomy not otherwise specified. Of those patients, treatment cohorts were stratified according to the surgical approach with an intention-to-treat paradigm: robotic $(n=223)$, laparoscopic $(n=1487)$, and open $(n=4717)$. Because information regarding the surgical approach was not uniformly provided before 2010, the sample was restricted to patients whose clinical information was added from 2010 to 2012 .

Analyses controlled for several patient level variables, including demographics (age, sex, race), income, education level, primary payer type (private, Medicaid, Medicare, other), and Charlson/Deyo score, which includes myocardial infarction, congestive heart failure, peripheral vascular disease, cerebrovascular disease, dementia, chronic pulmonary disease, rheumatologic disease, peptic ulcer disease, mild liver disease, diabetes, diabetes with chronic complications, hemiplegia or paraplegia, renal disease, moderate or severe liver disease, and AIDS [17]. Facilitylevel variables (type, geographic setting), tumor characteristics (American Joint Committee on Cancer clinical stage, clinical and pathologic TNM stage, lymph node invasion, and presence of metastases), extent of gastrectomy (proximal, distal, total, not otherwise specified), receipt of neoadjuvant chemotherapy, intraoperative and pathologic assessment of lymph nodes (lymph node removal, pathologist assessment of more than or fewer than 15 nodes, and pathologic findings of regional node positivity). and presence of distant metastases were also assessed. Outcomes of interest included presence of disease at surgical margins, adequacy of lymph node retrieval, postoperative LOS, and 30- and 90-day mortality, specifically as they related to the surgical technique.

\section{Statistical analyses}

Statistical analyses were used to determine differences in oncologic outcomes and predictors of postoperative LOS and mortality within 30 and 90 days of definitive surgery, stratified according to the surgical approach, and with the covariates described earlier being controlled for. Baseline patient, tumor, and surgery characteristics were compared between patients who underwent robotic, laparoscopic, or open gastrectomy by means of chi square tests for binary and categorical variables and $t$ tests for continuous variables. Univariate analysis was used to determine 
differences in surgical resection margins (positive or negative for cancer) and the adequacy of lymph node retrieval. Trends in use were compared between the surgical approaches for the 3-year timeframe of data collection. We reported means or proportions with associated $P$ values for comparison.

Linear regression was used to model the effects of baseline characteristics on postoperative LOS. Linear regression is a multivariate statistical approach used to fit a model for a continuous dependent outcome on a set of explanatory variables, and with the other regressors being controlled for [18]. We report coefficients with associated $95 \%$ confidence intervals and $P$ values. Logistic regression was used to model the effects of baseline characteristics on 30- and 90-day mortality. Like linear regression, logistic regression is a multivariate statistical approach used to fit a model for a binary dependent outcome on a set of explanatory variables, with other regressors being controlled for. We report odds ratios (ORs) with associated $95 \%$ confidence intervals and $P$ values. The goodness of fit of the logistic regression model was measured as the $C$ statistic.

Statistical significance for all analyses was defined as a $P$ value of less than 0.05 . All analyses were performed with Stata (version 12, StataCorp, College Station, TX, USA). This study was deemed to be exempt from institutional review board approval by the Human Subjects Protection Office of Penn State College of Medicine.

\section{Results}

\section{Patient characteristics}

Patients who underwent surgery for gastric cancer between 2010 and 2012 were included in the study. The analysis cohort included 6427 patients after exclusion of patients for whom data were missing or in whom no gastrectomy had been performed. Of the remaining patients, $223(3.5 \%)$ underwent robotic gastrectomy, 1487 (23.1\%) underwent laparoscopic gastrectomy, and 4717 (73.4\%) underwent open gastrectomy. Seventeen patients $(7.6 \%)$ who initially underwent robotic surgery were converted to open surgery and 282 patients (19\%) who initially underwent laparoscopic surgery were converted to open gastrectomy. The reasons for conversion were not available within the dataset.

Descriptive statistics are shown in Table 1. Patients who underwent robotic gastrectomy were more often white, privately insured, treated at academic facilities, and receiving care proportionally more often in the southeastern USA. Patients who underwent laparoscopic gastrectomy were more often white, privately insured or insured with Medicaid, treated at an academic facility, and received care in the northeastern USA. Patients who underwent open surgery were represented by greater proportions of individuals who were black, government insured, treated at nonacademic facilities, and received care proportionally more often in the south central USA.

Tumor and surgery statistics are shown in Table 2. Minimally invasive gastrectomy was more commonly performed in individuals undergoing proximal gastrectomy. Robotic surgery was more commonly performed in patients with stage 2 and stage 3 tumors, obtained more than 15 lymph nodes examined, and was performed in fewer individuals with metastatic disease. Laparoscopic surgery was more commonly performed in patients with earlier-stage disease and proportionally more often without lymph node removal. Open surgery was more commonly performed in patients who underwent total gastrectomy with more advanced disease, with concomitant lymphadenectomy of ultimately fewer than 15 nodes, with more node-positive and metastatic disease $(P<0.0001$, all). Among all patients undergoing gastrectomy for gastric cancer, the median LOS ranged from 8 to 9 days, with minimally invasive approaches requiring a shorter LOS of 8 days relative to the median LOS of 9 days required for patients undergoing open gastrectomy.

Assessment of trends in the use of the three surgical approaches found that although open gastrectomy is still far more commonly performed than minimally invasive gastrectomy, its incidence is declining, with corresponding increases in the use of both robotic and laparoscopic techniques. More specifically, open gastrectomies totaled 1678 in 2010 but declined to 1474 by 2012, whereas the use of laparoscopic approaches increased from 448 in 2010 to 549 in 2012 and the use of robotic approaches more than doubled from 46 in 2010 to 112 in 2012.

\section{Adequacy of oncologic resection}

The adequacy of oncologic resection, as it pertains to the presence of disease at resection margins and the number of lymph nodes ultimately examined, is shown in Table 3. Negative surgical margins were most commonly achieved in specimens resected robotically. Positive margins, either microscopically or macroscopically, were found most commonly in specimens resected via an open approach $(P<0.0001)$. The recommended 15 lymph nodes were resected and thereafter examined most commonly via robotic gastrectomy, with the recommended minimum nodal resection achieved less commonly via laparoscopic gastrectomy and least commonly via open gastrectomy $(P<0.0001)$.

In order to control for confounding, or mediating, variables, we performed multivariate analysis to eliminate as much selection bias as possible. When controlling for all 
Table 1 Demographic and facility characteristics of patients with gastric tumors, stratified by surgical approach

\begin{tabular}{|c|c|c|c|c|}
\hline Variable & Robotic $(n=223)$ & Laparoscopic $(n=1487)$ & Open $(n=4717)$ & $P$ \\
\hline Age (years) & 63.7 & 63.9 & 63.7 & 0.411 \\
\hline $18-40$ & $4.5 \%(10)$ & $3.6 \%(53)$ & $4.1 \%(193)$ & 0.288 \\
\hline $41-66$ & $53.8 \%(120)$ & $53.5 \%(796)$ & $52.3 \%(2468)$ & 0.182 \\
\hline $67-80$ & $37.7 \%(84)$ & $35.9 \%(534)$ & $36.2 \%(1708)$ & 0.920 \\
\hline$>80$ & $4.0 \%(9)$ & $7.0 \%(104)$ & $7.4 \%(348)$ & 0.364 \\
\hline Sex & & & & 0.694 \\
\hline Male & $70.4 \%(157)$ & $69.8 \%(1038)$ & $68.8 \%(3245)$ & \\
\hline Female & $29.6 \%(66)$ & $30.2 \%(449)$ & $31.2 \%(1472)$ & \\
\hline Race & & & & 0.018 \\
\hline White & $82.5 \%(184)$ & $82.6 \%(1228)$ & $80.7 \%(3808)$ & \\
\hline Black & $10.8 \%(24)$ & $10.5 \%(155)$ & $12.1 \%(573)$ & \\
\hline Other & $6.7 \%(15)$ & $6.9 \%(103)$ & $7.1 \%(336)$ & \\
\hline Payer type & & & & 0.049 \\
\hline Private & $47.1 \%(105)$ & $43.5 \%(647)$ & $41.4 \%(1955)$ & \\
\hline Medicaid & $2.7 \%(6)$ & $7.2 \%(107)$ & $7.0 \%(328)$ & \\
\hline Medicare & $47.5 \%(106)$ & $45.9 \%(682)$ & $46.9 \%(2211)$ & \\
\hline Other government & $0.4 \%(1)$ & $1.2 \%(18)$ & $1.4 \%(64)$ & \\
\hline None & $2.2 \%(5)$ & $2.2 \%(33)$ & $3.4 \%(159)$ & \\
\hline Median income (\$) & & & & 0.577 \\
\hline$<38,000$ & $14.8 \%(33)$ & $16.1 \%(240)$ & $16.6 \%(785)$ & \\
\hline $38,001-48,000$ & $21.1 \%(47)$ & $23.5 \%(350)$ & $23.9 \%(1126)$ & \\
\hline $48,001-63,000$ & $29.6 \%(66)$ & $24.9 \%(370)$ & $26.1 \%(1233)$ & \\
\hline 63,000 & $34.1 \%(76)$ & $34.1 \%(507)$ & $32.0 \%(1511)$ & \\
\hline No high school degree (\%) & & & & 0.167 \\
\hline$>21$ & $17.0 \%(38)$ & $16.5 \%(246)$ & $18.1 \%(856)$ & \\
\hline 13-20.9 & $26.5 \%(59)$ & $24.7 \%(367)$ & $27.2 \%(1281)$ & \\
\hline $7-12.9$ & $34.1 \%(76)$ & $32.3 \%(480)$ & $31.0 \%(1460)$ & \\
\hline$<7$ & $22.0 \%(49)$ & $25.2 \%(374)$ & $22.5 \%(1060)$ & \\
\hline Comorbidities & & & & 0.697 \\
\hline 0 & $70.0 \%(156)$ & $67.2 \%(999)$ & $67.8 \%(3196)$ & \\
\hline 1 & $24.2 \%(54)$ & $24.5 \%(364)$ & $24.7 \%(1165)$ & \\
\hline 2 & $5.8 \%(13)$ & $8.3 \%(124)$ & $7.5 \%(356)$ & \\
\hline Facility type & & & & $<0.0001$ \\
\hline Community & $3.1 \%(7)$ & $3.6 \%(54)$ & $5.4 \%(253)$ & \\
\hline Comprehensive community & $30.9 \%(69)$ & $31.1 \%(463)$ & $41.9 \%(1975)$ & \\
\hline Academic & $65.9 \%(147)$ & $65.2 \%(970)$ & $52.8 \%(2489)$ & \\
\hline Geographic location & & & & $<0.0001$ \\
\hline Northeast & $19.3 \%(43)$ & $30.1 \%(447)$ & $20.1 \%(949)$ & \\
\hline Southeast & $35.4 \%(79)$ & $20.0 \%(298)$ & $25.2 \%(1191)$ & \\
\hline North central & $21.1 \%(47)$ & $24.5 \%(365)$ & $24.3 \%(1145)$ & \\
\hline South central & $7.2 \%(16)$ & $9.1 \%(135)$ & $14.3 \%(673)$ & \\
\hline West & $17.0 \%(38)$ & $16.3 \%(242)$ & $16.1 \%(759)$ & \\
\hline
\end{tabular}

other covariates, we found the surgical approach did not influence the odds of achieving an $\mathrm{R} 0$ resection at a statistically significant level. In multivariate analysis of resection of at least 15 lymph nodes, however, both laparoscopic and robotic approaches were found to increase the odds of adequate lymphadenectomy. Use of a robotic approach provided a $51 \%$ greater odds of resection of at least 15 nodes (OR 1.51, $P=0.005$ ), whereas a laparoscopic approach provided a $16 \%$ greater odds (OR $1.16, P=0.023$ ) versus an open approach. 
Table 2 Tumor and surgery characteristics of patients with gastric tumors, stratified by surgical approach

\begin{tabular}{|c|c|c|c|c|}
\hline Variable & Robotic $(n=223)$ & Laparoscopic $(n=1487)$ & Open $(n=4717)$ & $P$ \\
\hline Gastrectomy & & & & $<0.0001$ \\
\hline Proximal & $65.0 \%(145)$ & $59.9 \%(890)$ & $49.2 \%(2320)$ & \\
\hline Distal & $6.7 \%(15)$ & $8.2 \%(122)$ & $11.8 \%(556)$ & \\
\hline Total & $26.5 \%(59)$ & $28.6 \%(425)$ & $36.1 \%$ & \\
\hline NOS & $1.8 \%(4)$ & $3.4 \%(50)$ & $2.9 \%(138)$ & \\
\hline Stage & & & & $<0.0001$ \\
\hline 0 & $2.2 \%(5)$ & $1.8 \%(27)$ & $1.2 \%(57)$ & 0.117 \\
\hline 1 & $30 \%(67)$ & $36.4 \%(542)$ & $29.4 \%(1387)$ & $<0.0001$ \\
\hline 2 & $32.7 \%(73)$ & $27.6 \%(411)$ & $31.0 \%$ & 0.034 \\
\hline 3 & $32.3 \%(72)$ & $29.7 \%(442)$ & $30.1 \%(1420)$ & 0.738 \\
\hline 4 & $2.2 \%(5)$ & $4.1 \%(61)$ & $8.0 \%(378)$ & $<0.0001$ \\
\hline Clinical $\mathrm{T}$ category & & & & $<0.0001$ \\
\hline $\mathrm{Tx}$ & $1.3 \%(3)$ & $1.3 \%(19)$ & $2.9 \%(138)$ & 0.001 \\
\hline T0, Tis, T1 & $19.7 \%(44)$ & $25.6 \%(381)$ & $21.4 \%(1009)$ & 0.002 \\
\hline $\mathrm{T} 2$ & $28.3 \%(63)$ & $24.0 \%(357)$ & $18.7 \%(880)$ & $<0.0001$ \\
\hline $\mathrm{T} 3$ & $45.3 \%(101)$ & $41.2 \%(612)$ & $43.2 \%(2039)$ & 0.279 \\
\hline $\mathrm{T} 4$ & $4.5 \%(10)$ & $7.4 \%(110)$ & $13.0 \%(613)$ & $<0.0001$ \\
\hline Pathologic $\mathrm{T}$ category & & & & $<0.0001$ \\
\hline $\mathrm{Tx}$ & $3.1 \%(7)$ & $1.9 \%(28)$ & $3.1 \%(148)$ & 0.039 \\
\hline T0, Tis, T1 & $38.1 \%(85)$ & $29.4 \%(437)$ & $24.1 \%(1135)$ & $<0.0001$ \\
\hline $\mathrm{T} 2$ & $14.8 \%(33)$ & $19.1 \%(284)$ & $13.6 \%(640)$ & $<0.0001$ \\
\hline $\mathrm{T} 3$ & $31.8 \%(71)$ & $35.4 \%(526)$ & $35.9 \%(1694)$ & 0.449 \\
\hline $\mathrm{T} 4$ & $9.4 \%(21)$ & $10.6 \%(158)$ & $18.7 \%(881)$ & $<0.0001$ \\
\hline Clinical N category & & & & 0.006 \\
\hline $\mathrm{Nx}$ & $1.3 \%(3)$ & $2.2 \%(33)$ & $2.4 \%(114)$ & 0.553 \\
\hline N0, N1 & $88.3 \%$ (197) & $85.7 \%(1275)$ & $82.6 \%(3896)$ & 0.002 \\
\hline $\mathrm{N} 2$ & $7.2 \%(16)$ & $7.7 \%(114)$ & $8.4 \%(395)$ & 0.589 \\
\hline N3 & $1.8 \%(4)$ & $3.7 \%(55)$ & $5.7 \%(268)$ & 0.001 \\
\hline Pathologic N category & & & & $<0.0001$ \\
\hline $\mathrm{Nx}$ & $3.6 \%(8)$ & $2.5 \%(37)$ & $2.9 \%(135)$ & 0.575 \\
\hline N0, N1 & $79.4 \%(177)$ & $68.1 \%(1013)$ & $61.3 \%(2893)$ & $<0.0001$ \\
\hline $\mathrm{N} 2$ & $7.2 \%(16)$ & $11.6 \%(172)$ & $13.7 \%(648)$ & 0.003 \\
\hline $\mathrm{N} 3$ & $6.3 \%(14)$ & $10.8 \%(160)$ & $15.8 \%(744)$ & $<0.0001$ \\
\hline Lymph node removal & & & & $<0.0001$ \\
\hline No & $9.0 \%(20)$ & $13.2 \%(196)$ & $7.6 \%(360)$ & \\
\hline Yes & $91 \%(203)$ & $86.8 \%(1291)$ & $92.4 \%(4357)$ & \\
\hline Lymph nodes examined & & & & $<0.0001$ \\
\hline 0 & $9.4 \%(21)$ & $13.4 \%(200)$ & $8.5 \%(400)$ & \\
\hline$<15$ & $32.7 \%(73)$ & $33.4 \%(496)$ & $41.7 \%(1965)$ & \\
\hline$>15$ & $57.8 \%(129)$ & $53.2 \%(791)$ & $49.9 \%(2352)$ & \\
\hline Lymph nodes positive & & & & $<0.0001$ \\
\hline 0 & $52.9 \%(118)$ & $47.3 \%(704)$ & $42.0 \%(1982)$ & \\
\hline $1-2$ & $23.3 \%(52)$ & $15.5 \%(230)$ & $17.2 \%(810)$ & \\
\hline $3-6$ & $7.6 \%(17)$ & $12.0 \%(179)$ & $14.4 \%(680)$ & \\
\hline $7-15$ & $5.4 \%(12)$ & $8.2 \%(122)$ & $12.2 \%(574)$ & \\
\hline$>15$ & $10.8 \%(24)$ & $16.9 \%(252)$ & $14.2 \%(671)$ & \\
\hline Metastasis & & & & $<0.0001$ \\
\hline Negative & $98.2 \%(219)$ & $96.4 \%(1434)$ & $92.8 \%(4376)$ & \\
\hline Positive & $1.8 \%(4)$ & $3.6 \%(53)$ & $7.2 \%(341)$ & \\
\hline
\end{tabular}

NOS not otherwise specified 
Table 3 Univariate analysis of oncologic resection outcomes among patients undergoing gastrectomy for gastric cancer, stratified by surgical approach

\begin{tabular}{lclll}
\hline Variable & Robotic $(n=223)$ & Laparoscopic $(n=1487)$ & Open $(n=4717)$ & $P$ \\
\hline Surgical margins & & & & $<0.0001$ \\
Negative & $94.2 \%(210)$ & $89.2 \%(1325)$ & $85.4 \%(4026)$ & \\
R1 & $3.6 \%(8)$ & $6.7 \%(100)$ & $7.5 \%(356)$ & \\
R2 & $0 \%(0)$ & $0.5 \%(7)$ & $0.7 \%(34)$ & \\
Involvement, NOS & $2.24 \%(5)$ & $3.6 \%(55)$ & $6.3 \%(301)$ & \\
Lymph nodes examined & & & $8.5 \%(400)$ & \\
0 & $9.4 \%(21)$ & $13.4 \%(200)$ & $417 \%(1965)$ & \\
$1-14$ & $32.7 \%(73)$ & $33.3 \%(496)$ & $49.9 \%(2352)$ & \\
$\geq 15$ & $57.8 \%(129)$ & $53.3 \%(791)$ & & \\
\hline
\end{tabular}

NOS not otherwise specified

\section{Perioperative outcomes}

The multivariate analysis for postoperative LOS is presented in Table 4. Linear regression showed that, relative to a reference patient who underwent open gastrectomy, use of a laparoscopic approach was associated with a LOS shorter by 1.08 days $(P<0.0001)$, whereas use of a robotic approach was not associated with a significantly lower LOS. Of note within this analysis, female sex $(-1.04$ days, $P<0.0001)$, nonwhite race (black, -0.93 days, $P=0.027$; other, -1.09 days, $P=0.028)$, treatment at a comprehensive community facility $(-0.68$ days, $P=0.020)$, treatment in a facility in the north central USA $(-0.95$ days, $P=0.013)$, and clinical T2 and T3 disease $(-1.35$ days, $P=0.002$, and -1.78 days, $P=0.001$ respectively) were associated with decreased LOS. Age of 67-80 years (1.61 days, $P=0.042$ ), having two comorbidities (1.30 days, $P=0.021)$, clinical stage 2 and stage 3 disease (3.06 and 3.21 days, $P=0.017$, both), and having lymph nodes surgically removed (4.18 days, $P<0.0001$ ) were associated with increased LOS.

The logistic regression results for 30- and 90-day mortality are shown in Table 5. Minimally invasive gastrectomy did not demonstrate any association with 30- or 90-day mortality relative to open gastrectomy. Age greater than 80 years (OR 6.12, $P=0.005$ ), having two comorbidities (OR 1.80, $P=0.011$ ), treatment at a community facility (OR 1.82, $P=0.034$ ), undergoing surgical lymph node removal (OR 3.94, $P=0.021$ ), and having more than 15 lymph nodes positive for disease (OR 4.64, $P=0.001$ ) were associated with increased 30-day mortality. Over a longer timeframe of 90 days, greater postoperative mortality was associated with age greater than 66 years (67-80 years, OR 3.60, $P=0.002$; greater than 80 years, OR 6.23, $P<0.0001$ ), having two comorbidities (OR 1.58, $P=0.010)$, treatment at a nonacademic medical facility (community, OR 1.58, $P=0.042$; comprehensive community, OR $1.35, P=0.015)$, having fewer than 15 lymph nodes pathologically examined (OR 2.89, $P=0.029)$, and having lymph-node-positive disease (1-2 nodes, OR 1.84, $P=0.001$; 7-15 nodes, OR 3.44, $P<0.0001$; more than 15 nodes, OR 4.91, $P<0.0001)$. Lower odds of mortality within 30 days postoperatively was associated with not receiving neoadjuvant chemotherapy (OR 0.58 , $P=0.010)$ and having proximal or distal, but not total, gastrectomy (OR 0.49, $P=0.047$, and OR 0.37 , $P=0.017$ respectively) relative to the reference patient. Having proximal, distal, or total gastrectomy (OR 0.47, $P=0.004$; OR $0.031, P<0.0001$; OR $0.51, P=0.010$ respectively) was associated with decreased 90-day mortality relative to the reference patient. $C$ statistics, measuring the goodness of fit for models of 30- and 90-day mortality, were 0.7694 and 0.7608 respectively.

\section{Discussion}

The emergence of minimally invasive surgery was a major step in the evolution of the field of surgical oncology [1]. In the setting of malignancy, where patients often come to surgery in a more fragile health state, much attention is paid to preservation of vitality and quality of life while balancing the necessity of adequate, and frequently morbid, oncologic resection. This balance has proven tenuous in an era of open surgery. With the implementation of laparoscopic and robotic techniques in the field of surgical oncology, there is greater potential for improved perioperative outcomes after adequate oncologic resections $[4,6$, 9, 16].

The present study sought to compare outcomes after minimally invasive gastrectomy with outcomes following open gastrectomy in a large US cohort of patients with surgically resectable gastric cancer. Oncologic resection, described by the presence of negative surgical margins and adequacy of lymph node retrieval, was found to be better when robotic and laparoscopic approaches were undertaken in appropriately selected patients. After other influential variables had been controlled for, a minimally invasive 
Table 4 Results of linear regression predicting the postoperative length of stay

\begin{tabular}{|c|c|c|c|c|}
\hline \multirow[t]{2}{*}{ Variable } & \multirow[t]{2}{*}{ Coefficient } & \multicolumn{2}{|c|}{$95 \%$ confidence limit } & \multirow[t]{2}{*}{$P$} \\
\hline & & Lower & Upper & \\
\hline \multicolumn{5}{|l|}{ Approach } \\
\hline Robotic & -0.86 & -2.20 & 0.48 & 0.206 \\
\hline Laparoscopic & -1.08 & -1.68 & -0.48 & $<0.0001$ \\
\hline Open & Reference & & & \\
\hline \multicolumn{5}{|l|}{ Age (years) } \\
\hline $18-40$ & Reference & & & \\
\hline $41-66$ & 1.08 & -0.28 & 2.44 & 0.119 \\
\hline $67-80$ & 1.61 & 0.06 & 3.17 & 0.042 \\
\hline$>80$ & 1.95 & -0.08 & 3.97 & 0.059 \\
\hline \multicolumn{5}{|l|}{ Sex } \\
\hline Male & Reference & & & \\
\hline Female & -1.04 & -1.60 & -0.48 & $<0.0001$ \\
\hline \multicolumn{5}{|l|}{ Race } \\
\hline White & Reference & & & \\
\hline Black & -0.93 & -1.75 & -0.10 & 0.027 \\
\hline Other & -1.09 & -2.06 & -0.12 & 0.028 \\
\hline \multicolumn{5}{|l|}{ Comorbidities } \\
\hline 0 & Reference & & & \\
\hline 1 & 0.22 & -0.03 & 0.85 & 0.484 \\
\hline 2 & 1.30 & 0.97 & 2.40 & 0.021 \\
\hline \multicolumn{5}{|l|}{ Facility type } \\
\hline Community & -0.77 & -1.95 & 0.41 & 0.200 \\
\hline Comprehensive community & -0.68 & -1.25 & -0.11 & 0.020 \\
\hline Academic & Reference & & & \\
\hline \multicolumn{5}{|l|}{ Geographic location } \\
\hline Northeast & Reference & & & \\
\hline Southeast & -0.75 & -1.51 & 0.01 & 0.052 \\
\hline North central & -0.95 & -1.70 & -0.20 & 0.013 \\
\hline South central & -0.65 & -1.56 & 0.26 & 0.161 \\
\hline West & -0.68 & -1.53 & 0.17 & 0.115 \\
\hline \multicolumn{5}{|l|}{ Stage } \\
\hline 0 & Reference & & & \\
\hline 1 & 1.15 & -1.02 & 3.33 & 0.299 \\
\hline 2 & 3.06 & 0.56 & 5.57 & 0.017 \\
\hline 3 & 3.21 & 0.58 & 5.84 & 0.017 \\
\hline 4 & 3.09 & -1.13 & 7.32 & 0.152 \\
\hline \multicolumn{5}{|l|}{ Clinical $\mathrm{T}$ category } \\
\hline Tx & -1.02 & -2.94 & 0.09 & 0.296 \\
\hline T0, Tis, T1 & Reference & & & \\
\hline $\mathrm{T} 2$ & -1.35 & -2.21 & -0.49 & 0.002 \\
\hline T3 & -1.78 & -2.86 & -0.69 & 0.001 \\
\hline $\mathrm{T} 4$ & -1.30 & -2.64 & 0.04 & 0.058 \\
\hline \multicolumn{5}{|l|}{ Lymph node removal } \\
\hline No & Reference & & & \\
\hline Yes & 0.45 & 0.25 & 0.65 & $<0.0001$ \\
\hline
\end{tabular}

The following covariates were controlled for but are not shown, secondary to lack of statistical significance: receipt of neoadjuvant chemotherapy, primary payer type, income, education level, extent of gastrectomy, American Joint Committee on Cancer clinical stage, pathologic $\mathrm{T}$ category, clinical and pathologic $\mathrm{N}$ category, pathologist assessment of more than or fewer than 15 nodes, pathologic findings of regional node positivity, and presence of distant metastases 
Table 5 Results of logistic regression predicting 30- and 90-day mortality

\begin{tabular}{|c|c|c|c|c|c|c|c|c|}
\hline \multirow[t]{3}{*}{ Variable } & \multicolumn{4}{|c|}{ 30-day mortality } & \multicolumn{4}{|c|}{ 90-day mortality } \\
\hline & \multirow[t]{2}{*}{ Odds ratio } & \multicolumn{2}{|c|}{$95 \%$ confidence limit } & \multirow[t]{2}{*}{$P$} & \multirow[t]{2}{*}{ Odds ratio } & \multicolumn{2}{|c|}{$95 \%$ confidence limit } & \multirow[t]{2}{*}{$P$} \\
\hline & & Lower & Upper & & & Lower & Upper & \\
\hline \multicolumn{9}{|l|}{ Approach } \\
\hline Robotic & 1.07 & 0.42 & 2.75 & 0.883 & 1.02 & 0.52 & 2.01 & 0.949 \\
\hline Laparoscopic & 0.89 & 0.59 & 1.33 & 0.572 & 0.83 & 0.62 & 1.12 & 0.226 \\
\hline Open & Reference & & & & Reference & & & \\
\hline \multicolumn{9}{|l|}{ Neoadjuvant treatment } \\
\hline None & 0.58 & 0.38 & 0.88 & 0.010 & 0.96 & 0.73 & 1.27 & 0.792 \\
\hline Neoadjuvant & Reference & & & & Reference & & & \\
\hline \multicolumn{9}{|l|}{ Age (years) } \\
\hline $18-40$ & Reference & & & & Reference & & & \\
\hline $41-66$ & 1.65 & 0.50 & 5.39 & 0.409 & 1.66 & 0.75 & 3.68 & 0.210 \\
\hline $67-80$ & 3.22 & 0.95 & 10.93 & 0.060 & 3.60 & 1.58 & 8.22 & 0.002 \\
\hline$>80$ & 6.12 & 1.72 & 21.76 & 0.005 & 6.23 & 2.60 & 14.90 & $<0.0001$ \\
\hline \multicolumn{9}{|l|}{ Comorbidities } \\
\hline 0 & Reference & & & & Reference & & & \\
\hline 1 & 1.04 & 0.72 & 1.48 & 0.851 & 1.14 & 0.88 & 1.47 & 0.321 \\
\hline 2 & 1.80 & 1.14 & 2.84 & 0.011 & 1.60 & 1.12 & 2.29 & 0.010 \\
\hline \multicolumn{9}{|l|}{ Facility type } \\
\hline Community & 1.82 & 1.04 & 3.15 & 0.034 & 1.58 & 1.02 & 2.46 & 0.042 \\
\hline Comprehensive community & 1.21 & 0.87 & 1.69 & 0.265 & 1.35 & 1.06 & 1.71 & 0.015 \\
\hline Academic & Reference & & & & Reference & & & \\
\hline \multicolumn{9}{|l|}{ Gastrectomy } \\
\hline Proximal & 0.49 & 0.24 & 0.99 & 0.047 & 0.47 & 0.28 & 0.79 & 0.004 \\
\hline Distal & 0.37 & 0.16 & 0.83 & 0.017 & 0.31 & 0.17 & 0.57 & $<0.0001$ \\
\hline Total & 0.55 & 0.27 & 1.14 & 0.107 & 0.51 & 0.30 & 0.85 & 0.010 \\
\hline NOS & Reference & & & & Reference & & & \\
\hline \multicolumn{9}{|l|}{ Pathologic $\mathrm{T}$ category } \\
\hline Treatment & 0.51 & 0.14 & 1.81 & 0.297 & 0.73 & 0.31 & 1.74 & 0.476 \\
\hline T0, Tis, T1 & Reference & & & & Reference & & & \\
\hline $\mathrm{T} 2$ & 0.58 & 0.30 & 1.10 & 0.092 & 0.64 & 0.40 & 1.00 & 0.050 \\
\hline $\mathrm{T} 3$ & 0.73 & 0.44 & 1.22 & 0.235 & 0.76 & 0.53 & 1.08 & 0.126 \\
\hline $\mathrm{T} 4$ & 1.26 & 0.70 & 2.26 & 0.445 & 1.29 & 0.84 & 1.99 & 0.244 \\
\hline \multicolumn{9}{|l|}{ Lymph node removal } \\
\hline No & Reference & & & & Reference & & & \\
\hline Yes & 3.94 & 1.23 & 12.65 & 0.021 & 1.80 & 0.76 & 4.26 & 0.180 \\
\hline \multicolumn{9}{|l|}{ Lymph nodes examined } \\
\hline 0 & Reference & & & & Reference & & & \\
\hline$<15$ & 1.77 & 0.47 & 6.57 & 0.397 & 2.89 & 1.11 & 7.52 & 0.029 \\
\hline$>15$ & 1.23 & 0.35 & 4.34 & 0.752 & 1.98 & 0.79 & 4.99 & 0.145 \\
\hline \multicolumn{9}{|l|}{ Lymph nodes positive } \\
\hline 0 & Reference & & & & Reference & & & \\
\hline $1-2$ & 1.42 & 0.88 & 2.30 & 0.152 & 1.84 & 1.30 & 2.60 & 0.001 \\
\hline $3-6$ & 2.13 & 0.83 & 5.45 & 0.114 & 1.81 & 0.91 & 3.59 & 0.092 \\
\hline $7-15$ & 2.11 & 0.84 & 5.31 & 0.114 & 3.44 & 1.80 & 6.56 & $<0.0001$ \\
\hline \multirow[t]{2}{*}{$>15$} & 4.64 & 1.80 & 11.94 & 0.001 & 4.91 & 2.51 & 9.61 & $<0.0001$ \\
\hline & \multicolumn{4}{|c|}{$\mathrm{AROC}=0.7694$} & $\mathrm{AROC}=0$ & & & \\
\hline
\end{tabular}

The following covariates were controlled for but are not shown, secondary to lack of statistical significance: sex, race, primary payer type, income, education level, geographic location, American Joint Committee on Cancer clinical stage, clinical T category, clinical and pathologic $\mathrm{N}$ category, and presence of distant metastases

$A R O C$ area under the receiver operating characteristic curve, NOS not otherwise specified 
technique was associated with greater odds of adequate lymph node resection, whereas neither laparoscopic nor robotic approaches increased the odds of R0 resections. A comparison of postoperative outcomes revealed that shorter LOS was associated with laparoscopic gastrectomy but that short-term mortality did not differ on the basis of the approach. Given trends in the USA in the use of minimally invasive approaches during the 3-year time course of data collection, it is possible that perioperative outcomes will improve after robotic and laparoscopic gastrectomy, with these perhaps ultimately outperforming conventional open gastrectomy in every metric. What remains to be determined is whether minimally invasive techniques impact long-term outcomes for US patients, such as survival.

In the USA, the standard of care for resectable advanced gastric cancer, as recommended on the basis of available scientific evidence and expert consensus, includes R0 resection and modified D2 lymph node dissection of 15 or more nodes [19]. Although patient selection has an important role in the feasibility of achieving an adequate oncologic resection and lymph node retrieval, a surgical approach was likewise found to be an important factor in the likelihood of resecting at least 15 nodes. Proponents of robotic technology, in particular, cite greater technical precision, more degrees of freedom in instrument articulation, and better visualization of anatomy relative to other surgical approaches [10]. With most of the experience and literature regarding surgery for gastric cancer coming from the East, previously published studies show a greater quantity of lymph nodes harvested during minimally invasive surgery, yet there is a general lack of consensus regarding improved $\mathrm{R} 0$ resection rates on the basis of the approach alone [11, 19-23]. For example, Vinuela et al. [24] performed a meta-analysis of all English-language studies since 1992, including several from Eastern centers, and found that when they compared open versus laparoscopic distal gastrectomy outcomes, the former were associated with better lymph node harvest quantitatively, but no difference was seen in the odds of having less than the recommended minimum of lymph nodes on the basis of the approach alone. With regard to R0 resection rates, Shen et al.'s [21] findings concurred with those of the present study. Perhaps the more clinically significant observation is that newer, minimally invasive technology is noninferior to the standard open procedure and potentially offers faster healing, fewer wound complications, and an earlier use of adjuvant therapy.

Our findings may be the first hint that the USA is beginning to observe the benefits of minimally invasive surgery for gastric cancer. Acknowledging the retrospective and observational nature of our study, we believe it still may provide some reassurance in light of previous studies from Western centers, in which minimally invasive approaches had not demonstrated clear superiority with regard to negative surgical margins and lymph node harvest [24-26]. Kelly et al. [26] examined oncologic outcomes after laparoscopic versus open gastrectomy at a single academic medical center and found a higher rate of $\mathrm{R} 1$ resection in laparoscopically resected specimens but no difference in the quantity of lymph nodes retrieved. Their single-institution case-control study compared patients treated as far back as 2005, a time when experience with minimally invasive gastrectomy was still limited in the USA, in contrast to the present study, which compares patients no farther back than 2010, treated at more than one oncologic facility. Although the present study's optimistic findings may also be explained by considering that patients intended for palliative resection may have disproportionately represented patients in the open surgery cohort, and therefore would not have been expected to have negative margins, the number of patients who would be characterized as having unresectable disease by oncologic standards (i.e., stage 4) was less than the number of patients found to have positive margins. Hence, this explanation likely does not wholly explain the worse margin status among patients undergoing open gastrectomy in univariate analysis. Lending further support to a temporal trend of improving outcomes is a multi-institution study by Coratti et al. [25], whereby robotic gastrectomy achieved adequate lymph node retrieval in $90 \%$ of patients over a 10-year period, with progressively better outcomes over time. No analysis was performed for the adequacy of oncologic resection in that time-trend analysis. The study by Coratti et al. [25] underscores the observation that outcomes may have generally improved as experience with minimally invasive gastrectomy has increased in the West.

We found in evaluation of perioperative outcomes among patients undergoing laparoscopic gastrectomy that LOS was decreased to a statistically significant level, but no statistically significant decrease in mortality was found. Our findings agree with the LOS benefit observed in both Eastern and Western studies, reflecting the well-known advantage of minimally invasive surgery. The finding of a 1.08-day decrease in LOS among patients undergoing laparoscopic gastrectomy in the present study was concordant with findings of previous studies, in which LOS was decreased by $0.8-5$ days postoperatively [21-24, 27 , 28]. The lack of statistical significance with regard to shortterm mortality may reflect the aggressiveness of this malignant disease, or perhaps alternatively, the physiologic trauma of gastric resection regardless of the technique. This is reflected in Vinuela et al.'s [24] meta-analysis and suggests that the surgical approach, or proficiency with it, has less influence on short-term mortality in gastric cancer, where tumor biology ultimately trumps treatment [29]. 
This is a retrospective analysis of tumor registry data, which carries some inherent limitations. First, our use of an intention-to-treat paradigm grouped individuals who ultimately received a laparotomy incision with those who underwent much less invasive procedures. Although this analytic method may have impacted outcomes, the outcomes from the small numbers of patients affected would likely not have changed the present study's statistically significant findings. Second, there are variables that were not available in the dataset that may be potential confounders and could not be controlled for. For example, the number of ports used for robotic or laparoscopic gastrectomy is not captured in the NCDB dataset, although it may modulate the relationships between the surgical approach and the adequacy of oncologic resection. Moreover, the operating surgeon's training, which might serve as a surrogate for proficiency, and volume in performance of gastric procedures for cancer is not documented within the NCDB. Given that gastrectomies in the USA might be performed by surgical oncologists, minimally invasive surgeons, and general surgeons alike, it is probable that patients undergoing minimally invasive gastrectomy are operated on by surgeons with greater experience and proficiency in performing oncologic gastric surgery, of any approach. Therefore, the benefits realized among these patients might be attributable to disproportionately greater experience among surgeons using laparoscopic or robotic methods. Still, given that minimally invasive surgery was a relatively novel modality for treatment of gastric cancer in the USA during the study period, surgeons performing minimally invasive gastrectomy still had to surmount a learning curve to achieve good outcomes [29]. As such, this learning curve may temper any influence proficiency has on the benefit seen for minimally invasive methods. Third, even in a clinical database in which trained nurse coders input data, there is always the potential for coding errors. However, it is probable that such errors would be neither abundant nor systematic. Fourth, the NCDB collects data from only oncologic centers in the USA that are accredited by the Commission on Cancer. Although various facilities do not contribute data because they lack Commission on Cancer accreditation, the NCDB's coverage of approximately $80 \%$ of cancer cases in the USA is likely representative of the US cancer population. Finally, although we controlled for a number of variables in multivariate analyses of outcomes, selection bias may persist as a result of variables not available for abstraction. This is particularly reinforced as we recognize statistically significant differences between the distribution of stage 4 disease patients undergoing resection by each of the three approaches. The acknowledgment of selection bias underscores the fact that in spite of newer technology and more advanced surgical techniques, evidence for or against a surgical approach must be balanced by the clinical judgment of treating clinicians and that conventional open surgery must remain in the armamentarium of those operating on gastric cancer patients.

We therefore conclude that within a US sample, minimally invasive gastrectomy is associated with decreased LOS and improved oncologic resections in patients deemed suitable for this surgical approach. Although the use of robotic and laparoscopic gastrectomy has increased since 2010 in the USA, we expect that the increase in use will continue as newer technologies and advanced surgical techniques pervade all corners of oncologic care in the USA. Further work remains to evaluate not only the longterm impact of minimally invasive gastrectomy for cancer but also the impact of other innovations in the care of gastric cancer patients to determine if US gastric cancer care may approximate that of patients treated in the East.

\section{Compliance with ethical standards}

Conflict of interest The authors declare that they have no conflict of interest.

Human rights statement All procedures followed were in accordance with the ethical standards of the responsible committee on human experimentation (institutional and national) and with the Helsinki Declaration of 1964 and later versions. No patient consent was necessary since this was a retrospective study.

\section{References}

1. Choh MS, Madura JA 2nd. The role of minimally invasive treatments in surgical oncology. Surg Clin N Am. 2009;89:53-77.

2. Kitano S, Iso Y, Moriyama M, Sugimachi K. Laparoscopy-assisted Billroth I gastrectomy. Surg Laparosc Endosc. 1994;4:146-8.

3. Hur H, Lee HY, Lee HJ, Kim MC, Hyung WJ, Park YK, et al. Efficacy of laparoscopic subtotal gastrectomy with D2 lymphadenectomy for locally advanced gastric cancer: the protocol of the KLASS-02 multicenter randomized controlled clinical trial. BMC Cancer. 2015; 15:355.

4. Kim HH, Han SU, Kim MC, Hyung WJ, Kim W, Lee HJ, et al. Prospective randomized controlled trial (phase III) to comparing laparoscopic distal gastrectomy with open distal gastrectomy for gastric adenocarcinoma (KLASS 01). J Korean Surg Soc. 2013;84:123-30.

5. Kim HH, Hyung WJ, Cho GS, Kim MC, Han SU, Kim W, et al. Morbidity and mortality of laparoscopic gastrectomy versus open gastrectomy for gastric cancer: an interim report-a phase III multicenter, prospective, randomized trial (KLASS trial). Ann Surg. 2010;251:417-20.

6. Kitano S, Shiraishi N, Uyama I, Sugihara K, Tanigawa N. A multicenter study on oncologic outcome of laparoscopic gastrectomy for early cancer in Japan. Ann Surg. 2007;245:68-72.

7. Terashima M, Tokunaga M, Tanizawa $\mathrm{Y}$, et al. Robotic surgery for gastric cancer. Gastric Cancer. 2015;18:449-57.

8. Son T, Hyung WJ. Robotic gastrectomy for gastric cancer. J Surg Oncol. 2015;112:271-8.

9. Diana M, Marescaux J. Robotic surgery. $\mathrm{Br} \quad \mathrm{J}$ Surg. 2015;102(2):e15-28. 
10. Parisi A, Nguyen NT, Reim D, Zhang S, Jiang ZW, Brower ST, et al. Current status of minimally invasive surgery for gastric cancer: a literature review to highlight studies limits. Int J Surg. 2015; 17:34-40.

11. Chen K, Xu XW, Zhang RC, Pan Y, Wu D, Mou YP. Systematic review and meta-analysis of laparoscopy-assisted and open total gastrectomy for gastric cancer. World J Gastroenterol. 2013;19:5365-76.

12. Lan H, Zhu N, Lan Y, Jin K, Teng L. Laparoscopic gastrectomy for gastric cancer in China: an overview. Hepatogastroenterology. 2015;62:234-9.

13. Xiong JJ, Nunes QM, Huang W, Tan CL, Ke NW, Xie SM, et al. Laparoscopic vs open total gastrectomy for gastric cancer: a meta-analysis. World J Gastroenterol. 2013;19:8114-32.

14. Kim W, Kim HH, Han SU, Kim MC, Hyung WJ, Ryu SW, et al. Decreased morbidity of laparoscopic distal gastrectomy compared with open distal gastrectomy for stage I gastric cancer: short-term outcomes from a multicenter randomized controlled trial (KLASS-01). Ann Surg. 2016;263:28-35.

15. Pugliese R, Maggioni D, Sansonna F, Costanzi A, Ferrari GC, Di Lernia S, et al. Subtotal gastrectomy with D2 dissection by minimally invasive surgery for distal adenocarcinoma of the stomach: results and 5-year survival. Surg Endosc. 2010;24:2594-602.

16. Kim HH, Han SU, Kim MC, Hyung WJ, Kim W, Lee HJ, et al. Long-term results of laparoscopic gastrectomy for gastric cancer: a large-scale case-control and case-matched Korean multicenter study. J Clin Oncol. 2014;32:627-33.

17. Deyo RA, Cherkin DC, Ciol MA. Adapting a clinical comorbidity index for use with ICD-9-CM administrative databases. J Clin Epidemiol. 1992;45:613-9.

18. Seltman HJ. Experimental design and analysis. 2015. http://www. stat.cmu.edu/hseltman/309/Book/Book.pdf. Accessed 2 Aug 2016.

19. National Comprehensive Cancer Network. NCCN clinical practice guidelines in oncology. Gastric cancer. 2015. Available via http://www.nccn.org/professionals/physician_gls/f_guidelines. asp. Accessed 10 Oct 2015.

20. Zeng YK, Yang ZL, Peng JS, Lin HS, Cai L. Laparoscopy-assisted versus open distal gastrectomy for early gastric cancer: evidence from randomized and nonrandomized clinical trials. Ann Surg. 2012;256:39-52.

21. Shen W, Xi H, Wei B, Cui J, Bian S, Zhang K, et al. Robotic versus laparoscopic gastrectomy for gastric cancer: comparison of short-term surgical outcomes. Surg Endosc. 2016;30(2):574-80.

22. Kwon IG, Cho I, Guner A, Kim HI, Noh SH, Hyung WJ. Minimally invasive surgery as a treatment option for gastric cancer in the elderly: comparison with open surgery for patients 80 years and older. Surg Endosc. 2015;29:2321-30.

23. Wu LM, Jiang XJ, Lin QF, Jian CX. Comparative study of clinical efficacy of laparoscopy-assisted radical gastrectomy versus open radical gastrectomy for advanced gastric cancer. Genet Mol Res. 2015;14:3459-65.

24. Vinuela EF, Gonen M, Brennan MF, Coit DG, Strong VE. Laparoscopic versus open distal gastrectomy for gastric cancer: a meta-analysis of randomized controlled trials and high-quality nonrandomized studies. Ann Surg. 2012;255:446-56.

25. Coratti A, Fernandes E, Lombardi A, Di Marino M, Annecchiarico M, Felicioni L, et al. Robot-assisted surgery for gastric carcinoma: five years follow-up and beyond: a single western center experience and long-term oncological outcomes. Eur $\mathbf{J}$ Surg Oncol. 2015;41:1106-13.

26. Kelly KJ, Selby L, Chou JF, et al. Laparoscopic versus open gastrectomy for gastric adenocarcinoma in the West: a casecontrol study. Ann Surg Oncol. 2015;22:3590-6.

27. Procopiuc L, Tudor S, Manuc M, Diculescu M, Vasilescu C. Open vs robotic radical gastrectomy for locally advanced gastric cancer. Int J Med Robot. 2015. doi:10.1002/rcs.1674.

28. Zong L, Seto Y, Aikou S, Takahashi T. Efficacy evaluation of subtotal and total gastrectomies in robotic surgery for gastric cancer compared with that in open and laparoscopic resections: a meta-analysis. PLoS One. 2014;9:e103312.

29. Zhou D, Quan Z, Wang J, Zhao M, Yang Y. Laparoscopic-assisted versus open distal gastrectomy with D2 lymph node resection for advanced gastric cancer: effect of learning curve on short-term outcomes. A meta-analysis. J Laparoendosc Adv Surg Tech A. 2014;24:139-50. 